\title{
The Risk Management Research of Treasure Futures
}

\section{Shuzhen Wang}

\author{
School of economics, Finance, Shanghai University, Shanghai, China
}

wshzh628@126.com

Keywords: Treasure future; Risk; Risk management; GARCH

\begin{abstract}
The futures as a derinatives in the financial market is not the real thing, but a standardization contract that can be trade. In reality, we can make use of spot and futures to hedge which can reduce risk, investors can do more or short to speculative, which can lead to the market turmoil. In the year of 1995 the event of " 327 " let the bond futures closed soon that just listed, after 17 years appear again. Many people hold different opinions about the futures, so the main idea of this paper is studies the risk of futures and risk management. First, we focus on the characteristics functions of treasure futures, and discusses the type and source of the risk of futures, second, treasure futures as object to establish a mathematical modle for the research of our country's futures risk. At last come to the conclusion and put forward feasible suggestions according to the domestic market.
\end{abstract}

\section{国债期货的风险管理研究 \\ 王淑真 \\ 上海大学 经济学院 金融学, 中国 上海 上海市 200444 \\ wshzh6280126. com}

摘要：期货作为金融市场上的衍生产品，不是实际存在的物，而是标准化可交易的合约。现 实中我们可以利用现货与期货进行对冲降低风险，投资者还可以做多做空进行投机，导致市 场的动荡。因此期货本身是存在风险的。1995 年的 “327” 事件让刚上市不久的国债期货关 闭了, 时隔 17 年之后又重新出现。好多人对期货的重新出现持有不同意见, 所以本文就是来 研究下期货的风险以及风险管理的问题。首先, 本文重点介绍了国债期货的特点、功能, 重 点论述期货风险的类型和来源, 之后以国债期货为研究对象建立数学模型研究我国期货的风 险, 最后, 得出结论并根据国内的行情提出可行性建议。

关键字：国债期货；风险；风险管理；GARCH

\section{1. 国债期货的概念及原理介绍}

1. 1. 概念

国债期货：是指通过交易场所预先确定价格并在未来一定的时间内进行交割的派生的交易方 式，属于金融期货的一种，是在金融市场不稳定的背景下首先在美国产生的。国债期货不是 实际的交割行为, 不会涉及到债券的所有权转移, 只是转让的价差; 必须在指定的交易场所 交割；合同都是标准化的，保证金制度实际是一种杜杆交易。

\section{2. 原理介绍}

无套利均衡是现代金融定价理论中的一个基本原理, 可以适用于任何资产的交易，即任何可以 套利的资产组合最终都会达到均衡状态，价格体现了内在价值。过去我国的国债期货之所以 会出现背离的事件就是因为脱离了基本原理, 出现了投机的机会造成了市场动荡, 极其容易 出现崩盘。现在国债期货的重新启动, 原理逐渐完善, 交易条件正在成熟, 定价原理更加完 善, 风险管理不断巩固。 
国债期货的价格理论上是通过 “一价定律” 和 “成本持有模型” 得出的, 简单的来说就是国 债期货的价格等于融资成本加上现券购入成本减去利息收入综合得出。

国债期货的交易策略也是会根据人们的不同目的而不同的，主要的就是在价格变动中低买高 卖的交易获得利差的投机策略，未来国债期货的价格也会随着基础资产现货的价格变动，此 时也会出现投机的行为，根据涨跌的方向不同还会出现多头投机和空头投机的行为；还有就 是利用不同商品之间不合理的价格关系，买进被低估的商品，卖出被高估的商品，在未来价 格重新回到体现内在价值的过程中获得价差收益的套利策略，此时又会出现跨市场、跨时期、 跨品种的套利行为; 国债期货最重要的作用就是套期保值, 做对冲用降低风险, 就是在现货 市场和期货市场对同一类商品同时买进相同数量但是方向相反的买卖行为, 或是通过不同的 投资比例是方差最小化

\section{2. 文献综述}

国债期货最早起源于美国，国外的期货市场很活跃、品种也很多。所以国外早就有很多对国 债期货的研究文献，而国内本来就发现的比较晚，外加 “327” 事件之后的停止，导致我国对 国债期货的研究更是少得可怜, 所以好多方面的研究只能参照国外的期货研究文献加上中国 特有的国情和股权结构特别的研究。之后的重新开始, 开启了国内对国债期货的新的研究, 因此也是众说纷纭，对开启国债期货的风险都持有不同的看法，也是各有各的道理。一下针 对国内外不同的研究做一个概述, 为本文奠定一定的基础。

\section{1. 国外文献综述}

Markowitz（1952）最早开始测度风险的，构建了著名的投资组合理论，并指出了分散投资的 重要性, 他认为资产之间存在不同程度的相关性并且相互影响, 相关性最少的组合可以有效 降低非系统风险（就是由各个企业之间独立存在的风险），还用表达式做了具体分析。Fama

（1965）在前人的研究基础上指出了大多数金融资产收益率具有 “尖峰厚尾” 的特征，不再 符合收益率呈正态分布的假设，资产收益的方差不能充分的代表波动性的大小。自从有了这 个发现，人们又纷纷证实了这个结论，为了处理这些特征 Engle(1982) 和 BoUerslev (1986) 分别提出了 $\mathrm{ARCH}$ 和 GARCH 模型, GARCH 模型是在 ARCH 模型上的延伸, 但是这些模型都需要 时间序列是平稳的、随机的、异方差的。虽然条件要求高但是很长一段时间内没法超越, 只 能延伸，所以接下来出现了一系列的 ARCH 族，这个方法也成为了经典。Jorion (1997) 在前 人的研究基础上给出了 $\mathrm{VaR}$ 权威的定义, 并对此做了推广, 分为动态的和静态的, 在 GARCH 模型的基础上能更准确的计算风险。后来人又在此基础上做了改进提出了 CVaR 模型, 指的是 超过 VaR 那部分的数值的平均损失，能刻画 VaR 之外的风险特征，也能解决正态分布假设下 的资产组合最优化。Su 和 Knowles (2010) 在测量风险的模型中又发现了混合正太模型得出的 结果会更精确。因此理论界的一些列研究风险的模型都出现的, 也为国内的研究打下了基础。 也为本文奠定了基础。

\section{2. 国内文献综述}

以上讲述的国外文献的研究路线，发展的时间长也有很多的使用价值，但是国内在长期关闭 的时间里都都选择了其他研究。所以我们一般是借助国外研究出来的方法, 借助我国所特有 的国内行情, 研究我国的发展, 下面就来讲讲国内的发展。吴晓求和应展宇 (2003) 运用具 体数据说明我国国债期货达到了巨大发展, 分析金融改革得出利率市场化的推进。姜洋 (2003) 的研究认为期货和衍生品市场没有融资功能, 但是提供了与融资功能不可分割的功能就是风 险管理, 提出了风险管理的重要性。蒋俊阳（2013）对国债期货的套利原理和定价原理做了 比较全面的分析与介绍, 为后来人发展研究做了理论基础。王玮和莫天瑜（2013）在 “新推 国债期货与 327 国债期货比较” 从不同的角度介绍了新推出的国债期货与以前的不同，说明 新推出的国债期货有了很多进步。 


\section{3. 国债期货概述}

3.1. 特点

从国债期货的定义我们可以看出他的特点有一些是与期货共有的特征，（1）国债期货只是转 移了与所有权有关的价格变化的风险，不会存在真正的所有权上的转移（2）国债期货的交易 地点必须是指定的, 遵循市场公开、交易自由的原则，禁止在场外交易或是私下对冲的行为

（3）裹在期货的特殊性就是他的标的合约 “名义标准债券” 或者也可以称之为 “虚拟债券” , 其实就是最终的价差交易，也是在保证金制度下的杜杆交易（4）他的交易实行的是没有负债 的每日结算制度（5）国债期货的交易很少出现实物交割的现象。

\section{2. 风险种类}

（1）信用风险：这是期货合约最主要的风险, 因为他本身就是一种合约，一种杜杆交易，最 终只是差价的交易而不需要真正的实物交割, 所以很容易因为人们的不履行约定的合约中的 条款而违约的风险，又可以分为交割前的风险和交割之后的风险（2）市场风险：这是金融产 品都不可避免的风险, 主要是因为投资者会因市场价格的波动出现的合约价值损失的风险, 这种风险可以通过强党的投资组合规避，但是国债期货本身具有更高的不确定性（3）流动性 风险：流动性越大风险越小（4）操纵风险：指在交易过程中，由于内部制度的不完善、或是 人为的有意无意的错误造成的损失, 也可能是操作人的失误造成的, 这种风险发生的概率比 较小（5）法律风险：法律更新的慢与金融市场的创新，导致一些交易没有法律可以依靠，人 们就可以利用法律的漏洞进行操控，给投资者造成的损失的风险。

3.3. 国债期货价格的影响因素

大体可以分为经济影响因素、政治影响因素、流动性影响因素、心理因素和其他因素。

经济因素又可以分为利率的影响, 国债期货以固定收益的国债为标的物, 固定了票面价值、 利率, 最后是固定收益, 当市场利率高于票面利率时我们可能会更少的选择国债, 此时就会 影响国债的价格; 通货膨胀也是影响价格的因素, 我们通常所公布的都是名义利率, 是带有 通货膨胀的, 如果通货膨胀很高就会带动名义利率升高, 可实际上的利率却没有提高, 因此 如果通货膨胀很高的化对价格的影响会很大, 我们会选择用实际利率; 经济增长带动整个市 场的价格变化也会带来影响。

政治因素主要是财政政策、货币政策、国际政治, 这些因素都会影响国家财政, 对通货膨胀 率、经济增长在不同程度上会有一些间接影响。流动性因素主要就是现货流动性和期货流动 性, 流动的越快反映在价格上的变化越大。心里因素主要是技术分析, 通过对基本面的分析 人们往往会给自己心理暗示, 而且这种暗示的作用会呈现放大的影响; 行为金融, 这是一门 专门解释人们心里的学科。

3.4. 国债期货的意义

国债期货本身就是一个利率的衍生品, 所以肯定会影响利率, 而且不是简单的只影响市场利 率, 他是整个利率体系的重要之处。作为成熟的市场经济, 利率是很关键的一块, 不管是货 币市场还是其他市场都会存在自己的利率，没有形成一个市场化的基准利率，而国债期货的 推出正好改变了这个状况。可以说国债期货是利率市场化的基石。

\section{4. 数据来源及模型建立}

\section{1. 数据来源}

数据都是来自于万德数据库。其中国债期货 TF1406 的数据是 2013 年 9 月 9 号到 2014 年 6 月 13 号的日数据，国债期货 TF1509 的数据到 2015 年 5, 股指期货 IF1506 的数据也是到 2015 年 5 月。用的是日度数据, 里面出去了缺失的数据。

4.2. 模型介绍

ARCH 模型：Engle (1982) 首先提出了 ARCH (自回归条件异方差) 模型。ARCH 模型的主要思想 是引入一个均值为 0 , 方差随时间变化且服从正态分布的扰动项扰动项的方差是一个自回归线 
性方程, 即扰动项方差服从 AR (q) 过程。

GARCH 模型: Bollerslev (1986) 在 ARCH 模型的基础上提出了 GARCH (p, q) 模型 (广义自回归条 件异方差模型)

GARCH 模型比 ARCH 模型有更好描述时间序列异方差特性和波动集聚效应, 同时 GARCH 模型具 有更为简洁的模型结构。金融资产的波动具有集聚效应, 当天的波动特性容易受近段时间内的 波动影响。

4.3. 实证分析

首先做了 TF1406 的收益率的折线图。从图中我们可以看出, 收益率是在 0 附近波动的, 但是 在 2013 年 11 月和 2014 年 5、6 月的时候出现了较大的波动, 说明国债期货还是存在风险的, 并且有时风险水平可能会超过预期或是平均水平很高。因此国债期货的风险也是不可忽视的。 接下来我们对国债期货和股指期货分别对成交量的变化、持仓量的变化、结算价、收盘价一 起做了折线图。对比分析发现股指期货的成交量逐年增加国债期货确实呈现波动性变动尤其 是去年的成交量变化呈现剧烈变动, 同时成交价也出现不同程度的变动。由此我们可以看出, 国债期货的重新出现还是存在一定的风险的, 并不是一些人想象的那么乐观, 即使经过了一 段时间的调整可还是存在很大的问题。所以我认为, 在中国研究国债期货的风险还是存在必 要的, 而且对风险管理的研究尤其重要。

接下来就是市政模型中用 GARCH 模型对风险的度量, 对于时间序列模型首先是要检验数据的 平稳性, 然后是自相关性, 最后是异方差性的特征性检验, 最后分析是否可以用 GARCH 模型 进行分析。

4. 3.1 单位根检验

采用 ADF 方法检验国债期货对数收益率的单位根, 检验结果如下

表 1 单位根检验

\begin{tabular}{cccc}
\hline & & $\mathrm{T}$ 值 & $\mathrm{P}$ \\
\hline \multirow{4}{*}{$\mathrm{ADF}$} & -12.4981 & 0 \\
& $1 \%$ & -4.00927 & \\
& $5 \%$ & -3.43471 & \\
& $10 \%$ & -3.14132 & \\
\hline
\end{tabular}

从表 1 中我们可以看到, ADF 值-12.4981 小于最小临界值-4.00, P 值近似于 0 拒绝存在单位 根的原假设, 接受备择假设, 所以收益率是平稳的, 符合 $\mathrm{GARCH}$ 模型对平稳性数据的要求。 4. 3.2 自相关检验

上面我们已经验证了数据的平稳性能, 证明数据是平稳的, 我们可以接下来验证自相关性了。 用 2013 年 9 月到 2014 年 5 月的 183 各观测做了自相关。我们可以看出 $\mathrm{P}$ 值都很大, 接受不 存在自相关的原假设, 因此收益率不具有自相关性, 是一群随机数据。符合了模型检验的随 机数据原则。

4.3.3 异方差检验

金融时间序列一般都具有异方差特性, 本文采用先将模型进行简单的自回归, 然后对残差项进 行 White 检验。本文进行了简单 1 阶自回归, 然后对残差项进行 White 检验, 检验结果如下 
表 2 异方差检验

\begin{tabular}{|c|c|c|c|}
\hline \multicolumn{3}{|c|}{ White 检验 } & \\
\hline F 统计量 & 20.61707 & $\mathrm{P}$ & 0 \\
\hline Obs*R-squared & 35.74541 & $\mathrm{P}$ & 0 \\
\hline
\end{tabular}

White 检验的 $\mathrm{F}$ 值 20.617, P 值 0, 拒绝原假设, 收益率具有异方差性, 因此符合 GARCH 模型 拟合时间序列，并在此基础上估计动态的 VaR。

4. 3. 4 GARCH 模型

表 3 GARCH 模型

\begin{tabular}{lrrrr}
\hline & 系数 & 标准差 & Z 统计量 & $P$ \\
\hline RETURN (-1) & -0.15012 & 0.042898 & -3.49935 & 0.0005 \\
\hline \multicolumn{5}{c}{ Variance Equation } \\
\hline C & 0.002755 & 0.001583 & 1.740393 & 0.0818 \\
$\operatorname{RESID~(-1)~} 2$ & 0.132182 & 0.059443 & 2.22366 & 0.0262 \\
$\operatorname{GARCH}(-1)$ & 0.80113 & 0.084239 & 9.510047 & 0 \\
$\operatorname{GARCH}(-2)$ & -0.12076 & 0.006329 & -19.08 & 0 \\
\hline
\end{tabular}

从表 3 可以看出条件方差方程中的常数项虽然显著, 但是取值数值太小, 为简化模型因此选择 没有常数项的条件方差, 为服从 $\mathrm{N}(0,1)$ 正态分布的随即项。从上面的图标可以求出模型的表 达式。

\section{5. 建议及结论}

通过运用图表分析法和 GARCH 模型对国债期货的风险的测量, 我们发现风险不仅是存在的, 而且还存在一定的聚类性, 波动性也会很大, 稍有不慎就可能造成不可挽回的损失, 国债 327 事件也在阐述了这样的事实。

从上面的分析可以看出国债期货的风险来源广泛，什么因素都可能会引起风险，虽然作为期 货如果恰到好处的话可以帮助投资者规避风险, 但本身也带来了特有的风险, 根据以上分析 的来源, 我们提出几条合理化建议:

(1) 我们应该合理的利用国债期货本身的特点帮助我们套期保值而不是进行投机行为, 我们 也可以借助模型测量风险以便于管理, 以此来减少风险的发生。(2) 我们建立公平的市场环 境，完善交易体系，建立完善的市场信息披露制度，增加市场透明度，规定上市公司的定期 披露制度, 避免市场被少数人控制 (3) 制定更多的市场监管体系, 对于大单的交易者我们要 特别注意, 同时规范中介机构的行为 (5) 完善公司规章制度、形成内部相互制约的监管体系, 完善交易系统，定期维护交易系统来规避不必要的风险。

\section{参考文献}

[1] Fama EF., The Behavior of Stock -Market Prices [J]., Journal of Business, （1965 ) 38:34-10.

[2] Jorion, P. Risk; Measuring the risk inValue-at-Risk [J]., Financial Analysis Journal， （1996） 11:47-56.

[3] Su E, Knowles, T W.:Measuring Bond Portfolio Value at Risk and Expected Shortfall in US Treasury Market[J].,Asia Pacific Management Review, (2010),15(4): 477-501. 
[4] Wu P T, Shieh S J: Value-at-Risk analysis for long-term interest rate futures: Fat-tail and long memory in return innovations [J]. Journal of Empirical Finance, (2007),14(2): 248-259.

[5] 吴晓求, 应展宇. 关于重新设立国债期货的若干问题 [J]. 财贸经济, (2003), 10:35-42.

[6]张晓菊, 曾光. 我国推出国债期货的可行性研究 [J]. 价格理论与实 践, 2008, No. 28301:65-66.

[7］赵秀峰. 国债期货仿真交易规则分析及建议 [J]. 中国证券期货, 2012, 03:62-63.

[8] 朱远. 运用利率期货实现风险管理 $[J]$. 商业时代, 2005, 2032

[9] 邱刚. 我国国债期货的风险管理研究 [J], 现代物业 (中旬刊), 2010, v. 9； No. 14001： $87-110$.

[10］王玮, 莫天新. 新推国债期货与 327 国债期货比较 [J] 金融市场研究, 2013. 11 VOL. 18: $102-109$

\section{Refenences}

[1] Fama EF, The Behavior of Stock -Market Prices [J]., Journal of Business, (1965)38:34-10.

[2] Orion, P. Risk; Measuring the risk in Value-at-Risk [J], Financial Analysis Journal, (1996) 11:47-56.

[3] Su E, Knowles, T W Measuring Bond Portfolio Value at Risk and Expected Shortfall in US Treasury Market[J].,Asia Pacific Management Review, (2010),15(4): 477-501.

[4] Wu P T, Shieh S J: Value-at-Risk analysis for long-term interest rate futures: Fat-tail and long memory in return innovations [J], Journal of Empirical Finance, (2007), 14(2): 248-259.

[5] Xiaoqiu Wu,Zhanyu Ying,Some problems about to set up a national debt futures[J],Finance and economic(2003)35-42

[6] Xiaoju Zhang,Guang Zeng,Feasibility study of present treasury futures[J],Price theory and practice, $(2008) 65-66$

[7] Xiufeng Zhao,Treasury futures simulation trading rules and suggestions,[J],China's securities and futures,(2012)62-63

[8] Yuan Zhu.Using interest rate futures implement risk management [J], Commercial Time, (2005)20-32.

[9] Gang Qiu,China's national debt futures risk management research[J],Modern property, (2010)87-110

[10] Wei Wang,Tianxin Mo,Compared with the 327 national debt futures push new Treasury futures,[J],The market research,(2013)102-109

作者简介：第一作者王淑真（1990）, 女, 河北省海兴县, 上海大学研究生, 主要研究金融 股票方向, E-mai1：wshzh628@126.com 